\title{
Concomitant therapies for varicose veins: Our high-volume, single-center experiences in the UK
}

\author{
Ahmed Elshiekh (D), Nick M Matharu (iD, Abhinav Vepa (iD) \\ Department of Surgery, University Hospitals Coventry and Warwickshire NHS Trust, Coventry, United Kingdom
}

\begin{abstract}
Objectives: This study aims to assess the feasibility of combined truncal endothermal ablation (ETA) and ultrasound-guided foam sclerotherapy (UGFS) in a single treatment session and compare this technique to published re-intervention rates following truncal ablation alone.

Patients and methods: The study was a retrospective data analysis of prospectively collected data. The patients had a preoperative US and then Endothermal Ablation (ETA) with concomitant UGFS. The need for a secondary treatment, technical success, operation time, patient satisfaction, complications, and recurrence were evaluated.
\end{abstract}

Results: The mean follow-up was $4.4 \pm 3.7$ months. The technical success was achieved in all patients. Sixty-six (93\%) of the patients did not need further treatments. Five patients (7\%) needed further interventions with repeated combined ETA and UGFS (n=1, 1.4\%) and sclerotherapy $(n=4,5.6 \%)$. These interventions did not increase the financial cost of treatment, compared to ETA alone.

Conclusion: Our study results suggest that one-stop dual therapy for the treatment of VV is associated with less need for further treatment, compared to truncal ETA alone without increasing the treatment cost.

Keywords: Endovenous therapy, varicose vein, venous ablation.

With 10 to $40 \%$ of the adult population being affected by a variable severity of varicose veins $(\mathrm{VVs})$, it is now one of the most encountered clinical presentations in the practice of vascular surgery. ${ }^{[1-3]}$ Varicose veins also represent a significant financial burden on the National Health Service with about $35,659 \mathrm{VV}$ surgeries performed in 2009/10. ${ }^{[4]}$

Various treatment options exist for the treatment of $\mathrm{VV}$, including topical medications, compression stockings, sclerotherapy, ablation therapy, and conventional surgery. ${ }^{[3-8]}$

The success rate of endothermal ablation (ETA) is reported to be as high as $90 \%$, while the success rates of ultrasound-guided foam sclerotherapy (UGFS) alone varies from 67 to $93 \% \cdot{ }^{[9-11]}$ However, ETA is not used to treat varicose tributaries and, thereby, requiring completion treatment at another episode in $21.5 \%$ of patients, and even up to $41.8 \%$ in some published studies. ${ }^{[12,13]}$

Combination therapy has been studied before. ${ }^{[14,15]}$ None of patients needed a repeat surgery, and less than $3 \%$ needed sclerotherapy at five years during follow-up in a randomized-controlled trial (RCT) comparing combination therapy for $\mathrm{VVs}$ in the form of highligation (HL) combined with either endovenous laser ablation (ELA) or stripping (S). ${ }^{[16]}$

Concomitant phlebotomy with ELA has been shown to decrease the need for secondary procedures by $62 \%$, compared to ELA alone, according to studies. ${ }^{[17,18]}$

Although the current evidence suggests lower recurrence rates and less need for repeated treatments in combination therapy, the practice in different 
centers still varies with no uniform agreement on combination therapy.

In the present study, we aimed to investigate whether single-episode, dual-modality treatment was associated with less need for repeated treatments in a British patient population.

\section{PATIENTS AND METHODS}

A retrospective cohort study design was used in which retrospective data analysis of prospectively collected data from the hospital records including operative notes, clinic letters, and theatre $\log$ data of a total of 71 patients (41 males, 30 females; median 63 years; IQ range 20) who underwent dualmodality VV treatment between January 2014 and December 2016 was carried out. All patients who had concomitant ETA and UGFS in the same treatment session were included, except for those who did not complete their follow-up or, those who did not have their data recorded in the hospital system. A written informed consent was obtained from each patient. The study protocol was approved by the local hospital research, development and innovation department. The study was conducted in accordance with the principles of the Declaration of Helsinki. All patients were initially admitted to the outpatient clinics following a general practitioner (GP) referral with symptomatic VVs. The patients were classified based on the CEAP (Clinical-Etiological-Anatomical-Pathophysiological) classification by visual inspection in the clinic. ${ }^{[5]}$

Duplex venous ultrasound scan was carried out in all patients preoperatively by our team of dedicated vascular technologists. The patients were, then, listed for ETA to long or short saphenous systems with concomitant UGFS. All procedures were carried out as a day case elective procedure under local anesthetic using $20 \mathrm{~mL}$ of $2 \%$ xylocaine with 1:200,000 adrenaline in $500 \mathrm{~mL} 0.9 \%$ normal saline per leg.

After antiseptic preparation, the patients were positioned either supine or prone in reverse Trendelenburg position where upon target varicosities were cannulated with 18 French (F) butterfly needles under the ultrasound guidance and secured in place.

The truncal vein was, then, cannulated with a standard Seldinger technique and $7 \mathrm{~F}$ sheath secured prior to the passage of a radiofrequency ablation (RFA) probe to a minimum safe distance from the confluence with the deep venous system (Covidien closure system; Covidien UK Co., Hampshire, UK).
Following tumescent anesthesia in the Trendelenburg position that the vein was ablated with direct visualization of the treated segment on Duplex scanning (Sonosite M-turbo, Fujifilm SonoSite Ltd., London, UK) and varicosities treated with 1:4 3\% sodium tetradecyl sulphate (STD) sclerosant for larger varicosities, $1 \%$ for small to medium ones (Fibrovein STD Pharmaceuticals, Hereford, UK).

Sclerotherapy sites were compressed and bandaged before fitting a class two compression stocking for 48 to $72 \mathrm{~h}$ and, then, revert to stockings. For two weeks, all patients were instructed to mobilize from day one to reduce chances of deep vein thrombosis.

Standard post-procedure follow-up was organized for the patients in the outpatient clinics for about 13 weeks where they were assessed. The need for a secondary treatment, technical success, operation time, patient satisfaction, complications, and recurrence were evaluated. The cost of the procedures was obtained from the financial department of the hospital.

\section{RESULTS}

A total of 71 patients had concomitant ETA and UGFS in the same treatment session. Demographic and clinical data of the patients presented in Table 1. The CEAP classification is shown in Figure 1.

The mean follow-up was $4.4 \pm 3.7$ months. Sixty-six (93\%) of the patients did not need any further treatment. Five patients (7\%), however, needed a further intervention with repeated combined ETA and UGFS $(n=1,1.4 \%)$ and UGFS $(n=4,5.6 \%)$.

Technical success which was defined as successful concomitant treatments in a single session was achieved in all patients. No intraoperative or immediate postoperative complications were encountered.

\begin{tabular}{|c|c|c|c|c|c|}
\hline & $\mathrm{n}$ & $\%$ & Mean $\pm S D$ & Median & IQ range \\
\hline Age (year) & & & & 63 & 20 \\
\hline \multicolumn{6}{|l|}{ Gender } \\
\hline Male & 41 & 58 & & & \\
\hline Female & 30 & 42 & & & \\
\hline \multicolumn{6}{|l|}{ Disease } \\
\hline Primary & 52 & 73 & & & \\
\hline Recurrent & 19 & 27 & & & \\
\hline \multicolumn{6}{|c|}{ Operation duration (min) } \\
\hline Unilateral & 63 & & $73 \pm 93$ & & \\
\hline Bilateral & 6 & & $115 \pm 50$ & & \\
\hline
\end{tabular}

SD: Standard deviation; IQ: Interquartile range. 
The mean operation time was $73 \mathrm{~min}$ for unilateral cases $(\mathrm{n}=65$, range, $40 \mathrm{~min}-145 \mathrm{~min})$ and $115 \mathrm{~min}$ ( $\mathrm{n}=6$, range, $89 \mathrm{~min}-162 \mathrm{~min}$ ) for bilateral cases. One operative list would typically include three concomitant procedures depending on the complexity of the cases.

All patients were adhered to the recommended compression stockings regimen.

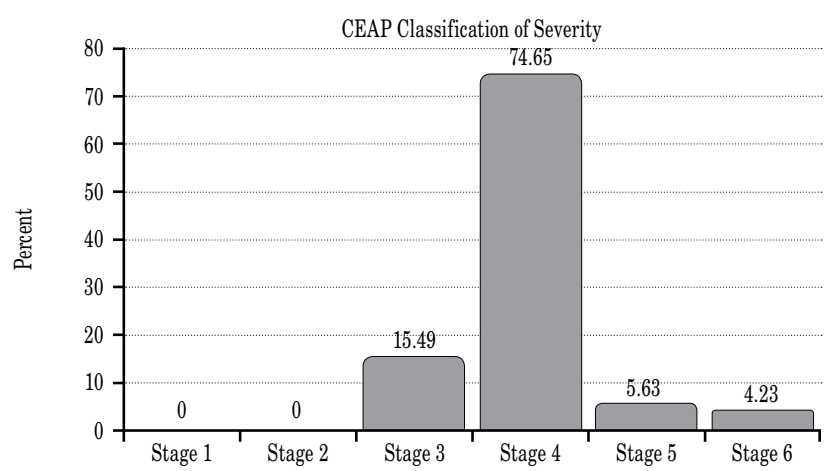

Figure 1. CEAP classification.

CEAP: Clinical-Etiological-Anatomical-Pathophysiological.

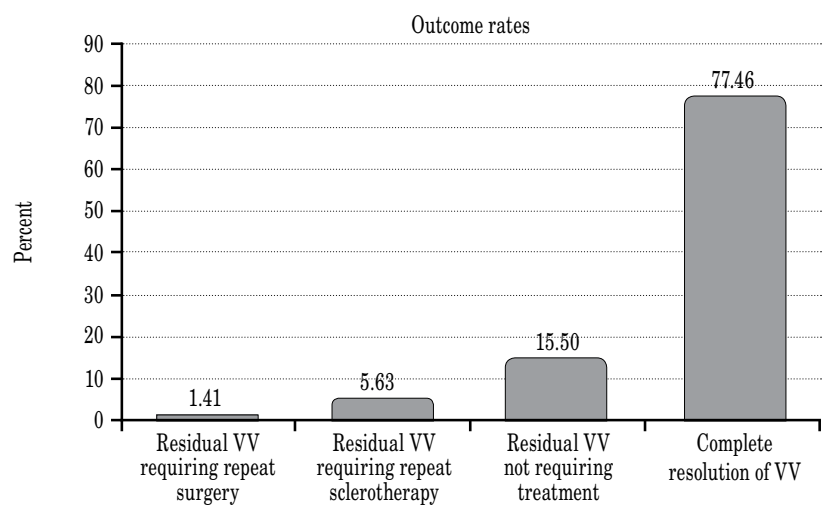

Figure 2. Summary of residual veins.

VV: Varicose vein.

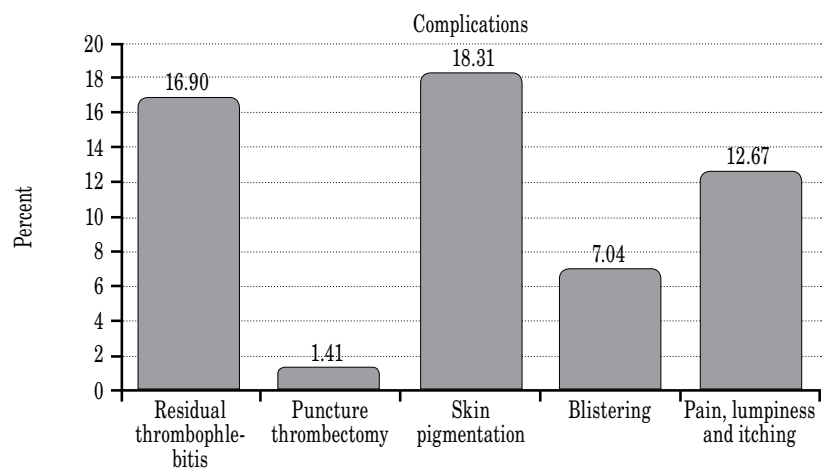

Figure 3. Postoperative complications.
All the patients included in our study were in CEAP Class $\geq 3$. Visual inspection in the clinic setting showed complete resolution of $\mathrm{VVs}$ in 55 patients (77.5\%). One patient (1.4\%) developed an ulcer and elected for conservative management. Five patients (7\%) needed further interventions. The remaining 10 patients (14\%) had minimal residual varicosities, but were satisfied with the overall result. A summary of the residual veins in the patients treated is shown in Figure 2.

The most common complications were skin pigmentation in 13 patients (18.3\%) with vein thickening (scarring) being the second most common in 12 patients (16.9\%). Only one patient needed puncture thrombectomy (1.4\%). Blistering was observed in five patients (7\%); however, this could be attributed to the compression pads used, which resolved, when the compression pads were changed. Five patients (7\%) experienced postoperative pain, lumpiness or itching. None of the patients had deep vein thrombosis or pulmonary embolism. Complications are summarized in Figure 3.

Total financial cost of a combined session of ETA and UGFS as a day case in our hospital was $£ 1,110$.

\section{DISCUSSION}

All the patients included in our study were in CEAP Class $\geq 3$ which can be attributed to the fact that only patients with advanced disease can be offered treatment in the UK in accordance with the Clinical commissioning groups (CCG) funding restrictions.

Despite treatment of truncal disease being the most important factor for preventing complication progression, patients attending for surgery often expect the visible target varicosities to be treated. Although UGFS and ETA are recommended as treatments by the National Institute for Health and Clinical Excellence, the former is limited by a maximum volume of foam that can be administered (typically $16 \mathrm{~mL}$ ), suggesting that patients often require multiple treatments. In general, ETA requires completion UGFS at another episode up to $41.8 \%{ }^{[13]}$ This is often inconvenient for patients, as most patients (71\%) prefer a one-stop service. ${ }^{[19]}$

Various combination modalities have been described in the literature. Endovenous laser ablation (EVLA) combined with UGFS was described in the literature with favorable results. ${ }^{[14]}$ The overall cost of RFA is less than that of EVLA with a tendency 
of less recurrence rate (11.6 vs. 7.3\%) according to a published RCT. ${ }^{[20]}$ The RFA ablation was shown to be the most cost-effective treatment for $\mathrm{VVs}$ over all other modalities including EVLA. ${ }^{[21]}$ The use of endovascular adhesives would drive the costs up and is not readily available in our institute. The RFA was chosen over EVLA as it is readily available in our institute, as well as it is technically easier requiring less personal protective equipment. Therefore, RFA was chosen over EVLA in this study.

The ability to perform the dual procedure in different patient populations, including those in whom general anesthesia may not be feasible, enabled us to adopt a total endovascular combining UGFS with ETA.

The costs of a combined session of ETA and UGFS as a day case in our hospital was $£ 1,110$. This did not exceed those of a single modality day case session of ETA, as there is not much difference in the use of the staff time, theatre space, or tools. Thus, combining the two procedures did not add much to the cost of treatment.

Although there were complications, none of them were life- or limb-threatening and they were all well-tolerated by the patients. Overall, we found that combination treatment could be offered as a single treatment episode much as the first-generation ligation, stripping, and avulsion used to be, but under local anesthetic, with 93\% success to avoid further treatment episodes. This finding is also consistent with the literature. ${ }^{[16]}$

Nonetheless, there are some limitations to this study. Our short follow-up is the main limitations which precludes long-term evaluation. In addition, the retrospective, non-randomized nature of the study reduces the quality of the data and may affect its generalizability. Therefore, further longer-term, randomized-controlled studies are needed to support our results.

In conclusion, our study results suggest that singleepisode truncal RFA and UGFS is a safe and effective treatment and is well accepted by the patients with cost-saving implications over staged treatment and is associated with less need for further treatment episodes. Based on these findings, we recommend one-stop dual therapy for the treatment of VVs.

\section{Declaration of conflicting interests}

The authors declared no conflicts of interest with respect to the authorship and/or publication of this article.

\section{Funding}

The authors received no financial support for the research and/or authorship of this article.

\section{REFERENCES}

1. Rabe E, Berboth G, Pannier F. Epidemiology of chronic venous diseases. Wien Med Wochenschr 2016;166:260-3. [Abstract]

2. Raju S, Neglén P. Clinical practice. Chronic venous insufficiency and varicose veins. $N$ Engl J Med 2009;360:2319-27.

3. Tisi PV. Varicose veins. BMJ Clin Evid 2011;2011. pii: 0212 .

4. National Institute for Health and Care Excellence (2013). Varicose veins: diagnosis and management. Nice Guideline 168. Available at: https://www.nice.org.uk/guidance/cg168 [Accessed: 01/10/2019]

5. Eklöf B, Rutherford RB, Bergan JJ, Carpentier PH, Gloviczki P, Kistner RL, et al. Revision of the CEAP classification for chronic venous disorders: consensus statement. J Vasc Surg 2004;40:1248-52.

6. Tassie E, Scotland G, Brittenden J, Cotton SC, Elders A, Campbell MK, et al. Cost-effectiveness of ultrasound-guided foam sclerotherapy, endovenous laser ablation or surgery as treatment for primary varicose veins from the randomized CLASS trial. Br J Surg 2014;101:1532-40.

7. Joseph N, B A, Faizan Thouseef M, Devi M U, Abna A, Juneja I. A multicenter review of epidemiology and management of varicose veins for national guidance. Ann Med Surg (Lond) 2016;8:21-7.

8. Venermo M, Saarinen J, Eskelinen E, Vähäaho S, Saarinen E, Railo M, et al. Randomized clinical trial comparing surgery, endovenous laser ablation and ultrasound-guided foam sclerotherapy for the treatment of great saphenous varicose veins. Br J Surg 2016;103:1438-44.

9. Mundy L, Merlin TL, Fitridge RA, Hiller JE. Systematic review of endovenous laser treatment for varicose veins. $\mathrm{Br}$ J Surg 2005;92:1189-94.

10. de Medeiros CA, Luccas GC. Comparison of endovenous treatment with an $810 \mathrm{~nm}$ laser versus conventional stripping of the great saphenous vein in patients with primary varicose veins. Dermatol Surg 2005;31:1685-94.

11. Jia X, Mowatt G, Burr JM, Cassar K, Cook J, Fraser C. Systematic review of foam sclerotherapy for varicose veins. Br J Surg 2007;94:925-36.

12. Disselhoff BC, der Kinderen DJ, Moll FL. Is there recanalization of the great saphenous vein 2 years after endovenous laser treatment? J Endovasc Ther 2005;12:731-8.

13. Meissner MH. What is effective care for varicose veins? Phlebology 2016;31:80-7.

14. Kalteis M, Adelsgruber P, Messie-Werndl S, Gangl O, Berger I. Five-year results of a randomized controlled trial comparing high ligation combined with endovenous laser ablation and stripping of the great saphenous vein. Dermatol Surg 2015;41:579-86. 
15. Lane TR, Onida S, Gohel MS, Franklin IJ, Davies AH. A systematic review and meta-analysis on the role of varicosity treatment in the context of truncal vein ablation. Phlebology 2015;30:516-24.

16. Yilmaz S, Ceken K, Alparslan A, Durmaz S, Sindel T. Endovenous laser ablation and concomitant foam sclerotherapy: experience in 504 patients. Cardiovasc Intervent Radiol 2012;35:1403-7.

17. Park SW, Yun IJ, Hwang JJ, Lee SA, Kim JS, Chang SH, et al. Fluoroscopy-guided endovenous foam sclerotherapy using a microcatheter in varicose tributaries followed by endovenous laser treatment of incompetent saphenous veins: technical feasibility and early results. Dermatol Surg 2009;35:804-12.

18. Carradice D, Mekako AI, Hatfield J, Chetter IC. Randomized clinical trial of concomitant or sequential phlebectomy after endovenous laser therapy for varicose veins. Br J Surg 2009;96:369-75.

19. Carroll C, Hummel S, Leaviss J, Ren S, Stevens JW, Cantrell A, et al. Systematic review, network meta-analysis and exploratory cost-effectiveness model of randomized trials of minimally invasive techniques versus surgery for varicose veins. Br J Surg 2014;101:1040-52.

20. Rasmussen LH, Lawaetz M, Bjoern L, Vennits B, Blemings A, Eklof B. Randomized clinical trial comparing endovenous laser ablation, radiofrequency ablation, foam sclerotherapy and surgical stripping for great saphenous varicose veins. Br J Surg 2011;98:1079-87.

21. Epstein D, Onida S, Bootun R, Ortega-Ortega M, Davies AH. Cost-Effectiveness of Current and Emerging Treatments of Varicose Veins. Value Health 2018;21:911-20. 